\title{
Polypropylene as an innovative substitute for jar material of horizontal axis, multi-jar ball milling machine to grind electrode materials for energy storage devices
}

\author{
Sourabh Shinde ${ }^{1 *}$, Taukir Momin ${ }^{2}$, Vispi Karkaria ${ }^{3}$ and Parshuram Karandikar ${ }^{4}$ \\ ${ }^{1}$ Dept. of Mechanical Engg., College of Engineering, Pune, India \\ ${ }^{2}$ Dept. of Mechanical Engg., College of Engineering, Pune, India \\ ${ }^{3}$ Dept. of Mechanical Engg., Northwestern University, IL, USA \\ ${ }^{4}$ Dept. of Electronics and Telecommunication Engg. Army Institute of Technology, Pune, India
}

\begin{abstract}
It is approximated that there will be a considerable amount of increase in worlds energy needs. So, there is a need of high energy storing devices when the load is low or moderate. Supercapacitors (SC) can be the competent options to serve this purpose. The paper recounts the performance of supercapacitors (SC) churned out from a horizontal axis multi-jar ball milling machine based on variations in material properties of ball milling jars. To store ample amount of energy, it is very essential to develop energy storing devices with large capacities and large ratings. The performance of energy storage devices is investigated using Manganese dioxide and Activated carbon as electrode materials. Majority of the times the granular size of raw-electrode materials is not enough fine to use them as electrodes directly. As a result of this, it is essential to crush the raw-electrode materials of the SC into fine powder. The energy storing capacity of SCs depends on size of particle, specific area, the of material and quantity to weight ratio of electrode material. In this paper an alternative material 'Polypropylene' is used as a substitute for Aluminum jars in horizontal axis multi-jar ball milling machine to crush the electrode materials. The effectiveness of both the jars is compared by evaluating the performance parameters of the SCs manufactured from electrode materials crushed in each of these jars. It is observed that the performance of SCs depends on type of ball milling machine, number of ball milling balls used, ball milling time, diameter of ball milling jars, speed of ball milling and material of ball mill jars properties. So, all other parameters are kept the same except the material of ball mill jars to scrutinize the effect of ball milling jars on performance of SCs.
\end{abstract}

Keywords: Ball Milling, Super-capacitors, activated carbon, Polypropylene, Manganese dioxide

\section{Introduction}

Energy storage devices can be possibly made of various materials like metal oxides, carbon and even with wood [1-5]. Electrode materials are the key element in determining the rating of an energy storage device [6]. Electrode materials should be crushed and grinded to fine powder for high effectiveness of the energy storage devices [7-9]. A lot of development has been observed in the field of energy storage devices for improving the effectiveness and capacity of these devices [10-16]. In today's era the developing advances in technology have also led to use of nanotechnology in the field of electrode material processing for energy storage devices $[17,18]$. Ionic bonds of electrolyte also play a vital role effectiveness of these energy storage devices $[19,20]$. There are two types of energy storing devices: Primary energy storing devices and secondary energy storing devices [21]. Primary cells cannot be recharged because chemical reaction in primary cells is irreversible and hence the primary cells cannot be reused. Example of primary cell is mercury cell. But on the other hand, the chemical reaction in secondary cells is reversible. As a result of this, secondary cells can be recharged. Supercapacitor (SC) is an example of secondary cell. Ball mill is a machine which can be used to grind these electrode materials into very fine powder [22,23]. Although this field of ball milling to grind electrodes is very untouched field and hence, not considered much in most of researches.

Super-Capacitors can typically store 10 to 100 times more energy per unit mass than electrolytic capacitors and has much higher charge acceptance and discharge rate [9]. SCs are used in applications where there is demand for fast charge and discharge cycles like in EVs, Photovoltaic systems, Ultra-batteries, defibrillators, etc. It is observed that good performance capability and charge storing capacity of the SC is result of the electrode material having surface area less than $3500 \mathrm{~m}^{2} / \mathrm{g}$, pore sizes in the range of $1-3 \mathrm{~nm}$ and pore volumes of $2.5 \mathrm{~cm}^{3} / \mathrm{g}$ [09]. Ball milling balls are very vital aspect to determine the effectiveness of ball mill machine. But it is observed that material properties of ball milling jars also play an

*Corresponding author: shindesr19.mech@coep.ac.in 
indispensable role in the performance of the ball milling. Although this area of research is not a much explored. So, the research paper tries to fill this gap in research with some concrete experimentations.

The purpose of this paper is organized as follows, Section: 2 consist of 2. Ball Milling of electrode material. Section: 3 consist of various possible jar materials and their properties used to make ball milling jars. Section: 4 shows the experimentation and results of the research. The section: 5 consist of conclusion of the experimentation. Section: 6 deals with the acknowledgement by the authors and Section: 7 deals with references reviewed.

\section{Ball Milling of electrode material}

There are conventional capacitors using dielectric materials between two electrodes which can bear high voltages because of which they have high voltage ratings [21]. SCs have two electrodes which are made of same materials. Electrolyte material is used which has high conductivity in between these two electrodes. Such high ionic liquids limit the resistance due to large number of ions. Basically, capacitance is decided by the specific surface area of electrode material. There are various types of SCs based on their construction: Rolled type and stacked type. In this case, stacked SCs is considered for research purpose because of the reason that, for laboratory experiments stacked type SCs are more suitable as no machinery is required in manufacturing of these type of SCs. In this research, SCs investigated are manufactured using a mixture of Manganese dioxide and activated carbon in the ratio of 1:1. The mixture of Manganese dioxide and activated carbon is applied in the proportion of $20 \mathrm{mg} / \mathrm{cm}^{2}$ [21] on a stainless-steel mesh using a brush. Considering $50 \%$ [21] of wastage factor in manufacturing of the SCs, $240 \mathrm{mg}$ of the electrode mixture is used. IPA is utilized to bind the Manganese dioxide and activated carbon mixture. Polyethylene is used as a dielectric between two electrodes [21]. Potassium sulphate of 0.65 molarity is employed as an electrolyte [21]. A DC supply of 2.2 Volts is forwarded to charge the SCs [09]. Most used electrode materials are polymers, metal oxides, carbon, and graphene. SC electrodes require high conductivity, to permit electrons to charge and discharge rapidly and high lithium-ion intercalation capacity, to allow for maximum charge storage capacity. Polymers for battery electrodes are made with the pertinent monomers and connectivity to meet these requirements. Several types of polymers such as polyaniline, polyacetylene, polythiophene, and poly(p-phenylene) are used as electrodes. Metal oxides as electrode materials have a large capacitance to volume ratio, which results into increase in energy density of capacitors, various metal oxides such as Manganese dioxide, $\mathrm{NiO}, \mathrm{Co} 3 \mathrm{O} 4, \mathrm{Fe} 2 \mathrm{O} 3$, $\mathrm{V} 2 \mathrm{O} 5$, etc. are intensively used as electrode materials [06]. Carbon because of its low cost and availability is the most common electrode material for SCs and batteries. Ball mill is a type of grinder used for crushing materials to transform them from granular form to powder form. It works on the principle of impact and enfeebled. Ball mill is quite effective but somewhat ignored field in production of nano-sized powder for electrode materials in energy storage materials like super-capacitors, batteries, and fuel cells. It consists of a hollow cylindrical jar rotating about its axis. The axis of the jar may be vertical or horizontal or even inclined at few degrees to the horizontal. Various types of ball milling machines are:

i. Vertical Axis ball milling machines

ii. Horizontal axis ball milling machines

iii. Planetary (Multi-jar) ball milling machines

iv. Industrial ball milling machines

v. Multi-jar ball milling machines

The paper describes the use of combination of horizontal axis and planetary ball milling machines termed as

'Horizontal axis multi jar ball milling machine'. Some important parts of this type of ball milling machine shown in Fig- 1 used for experimentation are:
a) Base Frame
b) Shafts
c) Timing pulleys
d) Timing belts
e) 100-watt, $300 \mathrm{rpm}$ Planetary DC gear motor
f) Ball milling jars
g) Jar holder
h) Controller to control and drive the motor, etc

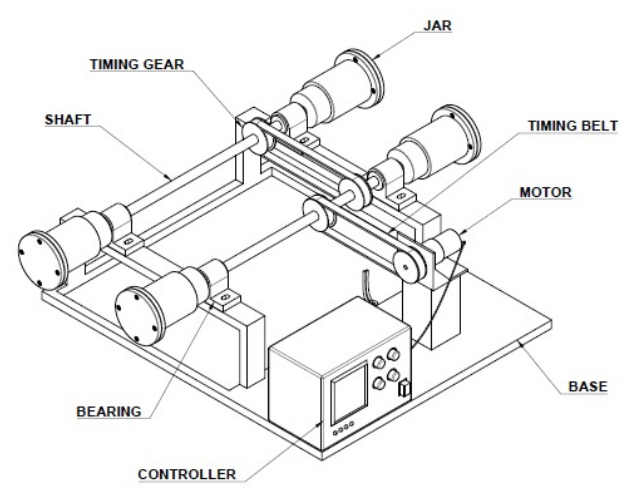

Fig 1- Construction of horizontal axis multi-jar ball milling machine

The advantages of this type of ball milling machine are:

a) The cost of installation is less as compared to other heavy ball milling machines.

b) The manufacturing cost of this ball milling unit is very low.

c) Operation cost is least possible.

d) It is suitable to grind for material of small quantity as well as large quantity, that means it is possible to fabricate ball mill machine of any capacity as per requirement.

e) This type of ball milling machine converts powder into extremely fine powder very effectively.

f) The machine produces minimum noise during operation.

Moreover, the horizontal axis multi-jar ball milling machine is perfect for laboratory experiments because of its low cost, light weight, and minimal operational noise. Further section deals with possible jar materials and their properties. This section also talks about the ball milling jar design. 


\section{Jar Materials}

A ball mill consists of a hollow cylindrical jar rotating about its own axis. The axis of the shell may be either horizontal or at a small angle to the horizontal or even vertical sometimes. It is partially filled with spherical balls. The grinding media are the balls, which may be made of stainless steel, ceramic, steel (chrome steel), or rubber. The hollow cylindrical jar is usually made of highgrade steel for large scale applications and Austenitic stainless-steel for small applications. Stainless steel ball mill balls are used in experimentation due to their easy availability. Sometimes Aluminum is used as a jar material when grinding with soft balls (Made of rubber, etc.). Austenitic stainless-steel has an Ultimate tensile strength of $515 \mathrm{MPa}$ whereas, its density is as high as $7850 \mathrm{~kg} / \mathrm{m}^{3}$. The microstructure of austenitic stainlesssteel is composed of columnar grains with dendritic or cellular sub-structure. Aluminum is a metal having density less than many other common metals. Aluminum is a soft, silvery metal with a face-centered cubic crystal structure (FCC), a feature of ductile metals. Aluminum has an Ultimate tensile strength of $180-290 \mathrm{MPa}$ depending on the type of alloy and has a moderate density of $2700 \mathrm{~kg} / \mathrm{m}^{3}$. Polypropylene (PP) is used as a jar material in the research. Polypropylene (PP) possesses an ultimate tensile strength of $80 \mathrm{MPa}$ at most but the density of the material is $910 \mathrm{~kg} / \mathrm{m}^{3}$. Polypropylene is produced by chain-growth polymerization from the monomer propylene. Polypropylene is a member of the group of polyolefins and is partially crystalline and non-polar. Its properties are like polyethylene, but it is moderately harder and more heat resistant. Moreover, Polypropylene can be produced in various colors thereby providing an aesthetic look to the ball milling jars and hence making ball milling machine aesthetically pleasing. Polypropylene also has excellent sound energy damping and vibration damping characteristics. As a result, of all these characteristics polypropylene is selected as a jar material in this experiment.

The cost of manufacturing of polypropylene is quite high as compared to Aluminum and Austenitic Stainless-steel because of its low thermal capability. But when machined at appropriate speed at around 200-400 rpm polypropylene can be turned into required shape and size. The manufacturing cost incurred for manufacturing the jars of Aluminum and Polypropylene is approximately $13.47 \$$ and $29.63 \$$ per jar respectively. As, the turning of Polypropylene requires highly skilled technicians. Polypropylene must be turned at not more than $400 \mathrm{rpm}$ keeping very appropriate depth of cut. Table- 1 shows the comparison between properties and cost considerations of Austenitic stainless-steel, Aluminum and Polypropylene.

Table 1-Material properties and material cost

\begin{tabular}{|c|c|c|c|}
\hline Material & $\begin{array}{c}\text { Ultimate } \\
\text { Tensile } \\
\text { Strength }\end{array}$ & Density & Material Cost \\
\hline $\begin{array}{c}\text { Austenitic } \\
\text { Stainless-Steel }\end{array}$ & $515 \mathrm{MPa}$ & $\begin{array}{c}7850 \\
\mathrm{~kg} / \mathrm{m}^{3}\end{array}$ & $2.02 \$ / \mathrm{Kg}$ \\
\hline
\end{tabular}

\begin{tabular}{|c|c|c|c|}
\hline Aluminum & $290 \mathrm{MPa}$ & $\begin{array}{c}2700 \\
\mathrm{~kg} / \mathrm{m}^{3}\end{array}$ & $1.95 \$ / \mathrm{Kg}$ \\
\hline Polypropylene & $80 \mathrm{MPa}$ & $\begin{array}{c}910 \\
\mathrm{~kg} / \mathrm{m}^{3}\end{array}$ & $2.16 \$ / \mathrm{Kg}$ \\
\hline
\end{tabular}

The ball milling jars are manufactured as shown in the Fig below:

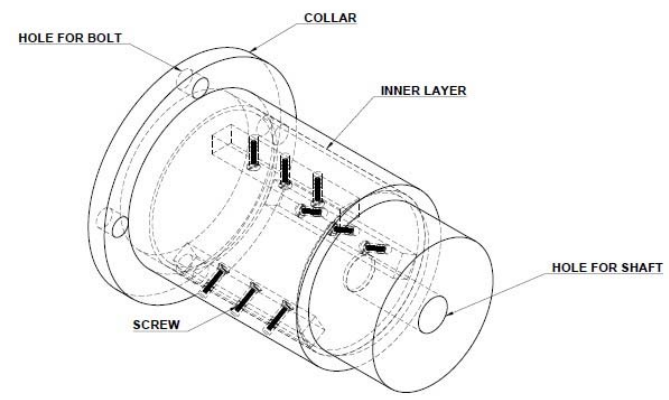

Fig 2- Ball milling jars for horizontal axis multi jar ball milling machine

Aluminum jars are heavy due to their high density and hence consume more energy by applying increased load on motor. Moreover, Aluminum jars produce a very heavy noise during operation and their leakage is ungovernable. So, there is a need for an alternative material which can overcome all these drawbacks and be a boon to SC or energy storage developing companies.

\section{Experimentation and Results}

SCs are made using a mixture of Manganese dioxide and activated carbon in the proportion of $1: 1$ by weight. At first Manganese dioxide is grinded in Horizontal axis multi-jar ball milling machine. Two samples of manganese dioxide are obtained each grinded in Aluminium jar and Polypropylene jar. Then the mixture of grinded Manganese dioxide and activated carbon is bind together using IPA solution. A slurry of grinded Manganese dioxide and activated carbon is formed. This mixture is applied over stainless-steel mesh using a paint brush in the proportion of $20 \mathrm{mg} / \mathrm{cm}^{2} .50 \%$ of wastage factor is considered while applying the slurry and hence $240 \mathrm{mg}$ of slurry is applied over stainless-steel mesh. Two such electrodes are made, and they are allowed to dry for some time. Then Polyethylene is used as a dielectric separator between these two electrodes. The Polyethylene sheets are pasted together using an epoxy.

The SC is packed and pressed tightly so as epoxies can hold the polyethylene sheets together firmly. Now, an electrolytic solution of Potassium sulphate and Water is prepared maintaining a molarity of 0.65 . The capacitors are of two types based on construction: Stacked type and rolled type. Based on electrolytic solution used supercapacitors can be classified as: aqueous and nonaqueous electrolytic supercapacitors. The supercapacitor discussed in this paper is a stacked type of supercapacitor which uses aqueous electrolytic solution. This type of supercapacitor is used due to its low cost and manufacturing ease. 


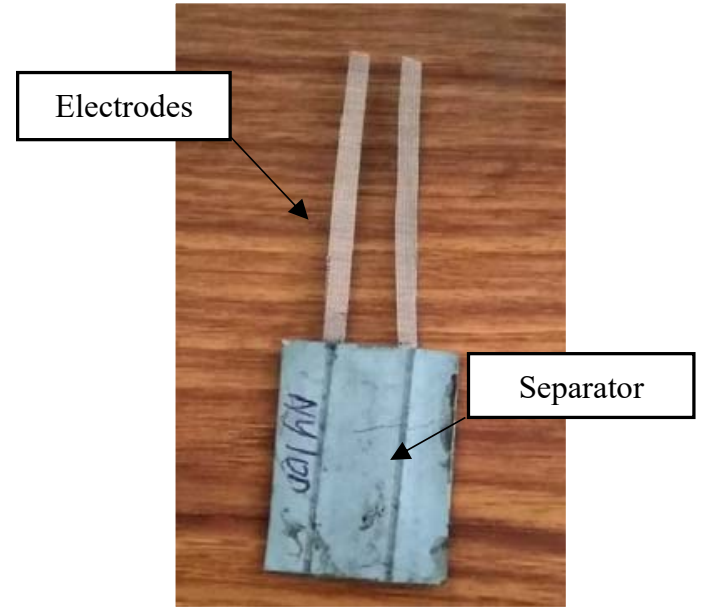

Fig 3- Constructional image of stacked type Supercapacitors

The performance analysis of the ball milling jars can also be done using an electron beam microscope. In electron beam microscope a stream of high voltage electrons (usually $5-100 \mathrm{KeV}$ ) is generated by the Electron Source (usually a heated tungsten or field emission filament) and accelerated in a vacuum towards the object using a positive electrical potential. This stream is limited and focused using metal holes and magnetic lenses into a thin, focused, monochromatic beam. This beam is focused onto the sample using a magnetic lens. Interactions occur inside the irradiated sample, affecting the electron beam. These interactions and effects are detected and presented into an image. The magnifying capacity of an electron beam microscope can be as high as 400-2000 times. These microscopes can be used to measure the fineness of ball milled products. But these microscopes are very delicate and expensive to work with.

So, an indirect approach is used to analyse the performance of proposed ball mill jars. The electrode materials are grinded in the horizontal axis multi-jar ball milling machine and SCs are manufactured using the electrode materials grinded in these jars. The capacitance rating of these capacitors is calculated by chargedischarge tests. In this research, indirect approach becomes more beneficial than direct approach as indirect approach directly links the application-based analysis of proposed ball mill machine jars and moreover, indirect approach is cost effective and easy to apply. Jars made of two materials viz. Aluminium, and Polypropylene are compared in this paper. This comparison is done by evaluating the capacitance rating of the SCs manufactured by electrode materials processed in these two jars.

The jars of horizontal axis multi-jar ball milling machine are rotated at $80 \mathrm{rpm}$ with a cycle time of 20 hours. The appropriate speed of ball milling is very essential as more speed will lead to excessive centrifugal forces and hence material will stick to the inner walls of jar during ball milling and hence the effectiveness of the process will be reduced. But on the other hand, if the speed of ball milling is too low very less centrifugal forces will be generated so the material to ball mill will experience lesser impact and attrition.
Therefore, based on observations appropriate rotational speed is found out to be $80 \mathrm{rpm}$. Charge discharge tests are done with five minutes as a standard discharge time for both the SCs made from electrodes processed in both Aluminium and Polypropylene jars. The capacitance rating is calculated, and it is observed that SCs made of the electrode material processed in polypropylene jars shows higher capacitance rating as compared to SCs made of electrode material processed in Aluminium jars.

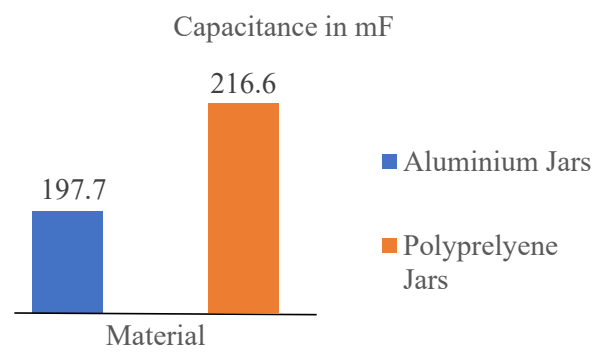

Fig 4- Capacitance with jar of Polypropylene material

Fig- 4 depicts the capacitance variation for electrode materials processed in Aluminium jars and polypropylene jars respectively. Variation of almost 18.8 millifarad is observed. An observation is made that the density of jar material is inversely proportional to the rate of capacitance of the SCs produced from electrode materials processed in these jars. Performance evaluation of these SCs produced from electrode materials crushed in Aluminium and Polypropylene jars respectively is carried out and results are obtained as shown in the charts below:

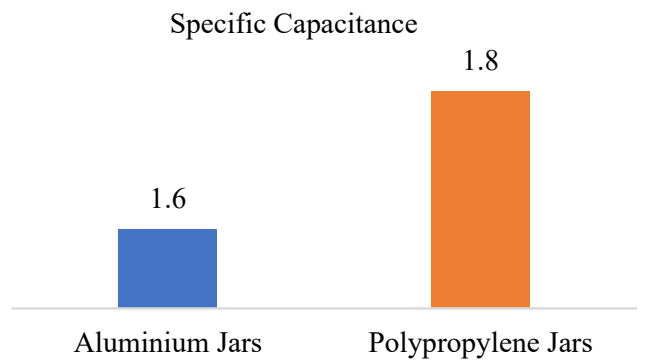

Fig 5- Specific Capacitance with jar of Polypropylene material

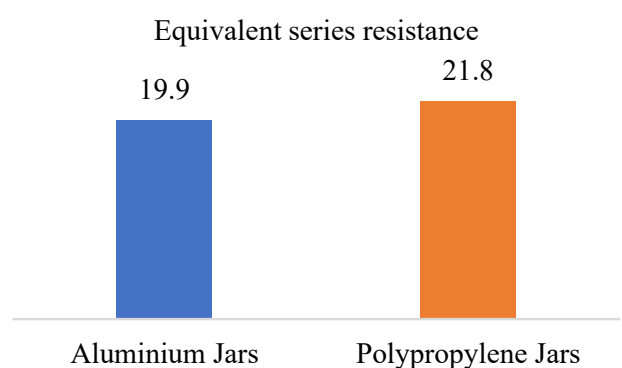

Fig 6- Equivalent Series Resistance with jar of Polypropylene material 


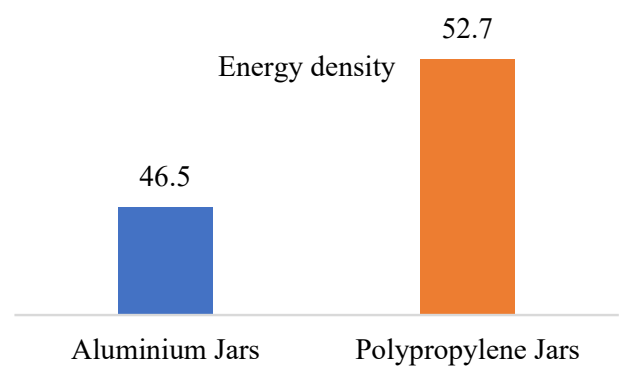

Fig 7- Energy Density with jar of Polypropylene material

Fig- 4 shows the chart depicting the experimental values of capacitance in millifarad. It can be observed that a capacitance increase of 18.9 millifarad is registered. Specific capacitance and energy density (Fig- 5 and Fig7 ) is increased by approximately $12 \%$ by substituting Polypropylene as a jar material for horizontal axis, multilayer ball milling machine. The equivalent series resistance (Fig- 6) in both the cases is almost equal with a minor difference of just 1.9 Ohms.

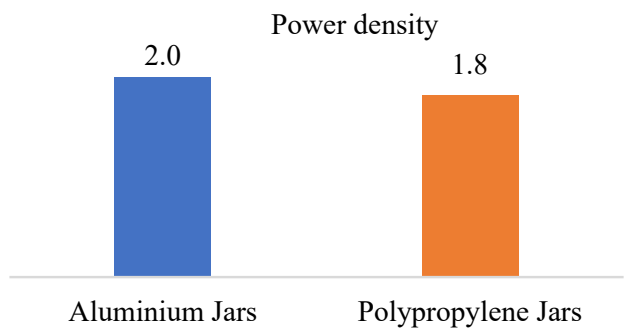

Fig 8- Power Density with jar of Polypropylene material

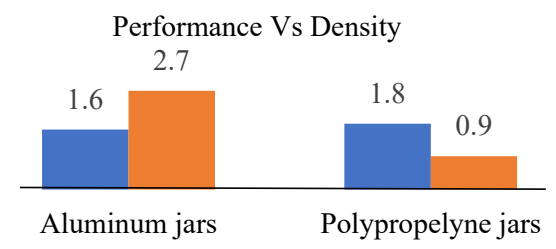

- Sp. Capacitence(F/g) $\square$ Material Density $\left(\mathrm{g} / \mathrm{cm}^{\wedge} 3\right)$

Fig 9- Correlation of specific capacitance with jar material mass density

Based on observations it is investigated that if the density of jar material is reduced by $33.7 \%$ the performance of the SCs manufactured from electrode materials processed in these jars is increased by $10 \%$ approximately. So, overall performance parameters of electrodes manufactured in jars made of Polypropylene show better results than the electrode produced from jars made of Aluminium.

\section{Conclusions}

The research investigates the effect of material of ball mill jars on the performance of ball milling machine. An indirect approach is represented in the paper, where ball milled, material is used to make electrodes for SCs and then the performance of SCs is evaluated and results for two jar materials viz. Aluminium and Polypropylene are compared. A horizontal axis multi jar ball milling machine is employed in this research for experimental purpose. The ball milling is done for 20 hours and at rotational speed of about $80 \mathrm{rpm}$. After the experiments it observed that the SCs made from Manganese dioxide and activated carbon grinded in polypropylene jars using stainless steel ball mill balls show a better capacitance rating. Moreover, by use of polypropylene few more advantages were observed, they are as follows:

a) The overall weight of the ball milling jars is reduced by $69 \%$ as Polypropylene is light than Aluminium

b) Polypropylene jars produce $23 \%$ less noise while in operation as Polypropylene is composite and composite materials absorb sound.

c) The power required to ball mill is reduced by $20 \%$ as using Polypropylene jars the overall weight of the machine is reduced. Hence, the load on motor is also reduced.

d) Polypropylene is available in various colours so aesthetic look of the machine is modified.

e) Cleaning of the jars can be effective. As the jar colour can be used in contrast with colour of material to grind.

f) It is also observed that the polypropylene jars produced $60 \%$ less vibrations and hence leakages were also reduced as composites have better vibration damping abilities.

Thus, it can be concluded that polypropylene can be a sublime alternative to Aluminium for horizontal axis multi-jar ball milling machine or any other small scale ball milling machine. So, it will provide a great alternative to manufacturers of SCs to allow them to accompany Polypropylene as a jar material and reduce the overall weight of the machine and decreasing the load on motor considerably.

\section{Acknowledgement}

The authors of this paper gratefully acknowledge the support of principal and management of Army Institute of technology, Pune for providing laboratory facility during the experimentation. The also express their gratitude towards the principal and staff of College of Engineering, Pune for their help in the preparation of this paper. Last but not the least, authors also express their thankfulness towards the workshop staff of Army Institute of Technology for helping and guiding them wherever and whenever needed.

\section{References}

1. Yu Ma, Jinwei Yin, Hanqin Liang, Dongxu Yao, Yongfeng Xia, Kaihui Zuo, Yu-ping Zeng. "A twostep approach for making super capacitors from 
waste wood". J. Clean. Prod. January 2021, Vol. 279, (123786).

2. Li Zang, Dandan Wu, Gaowei Wang, Yongtai Xu, Hongxia Li, Xingbin Yan. "An aqueous zinc-ion hybrid super-capacitor for achieving ultrahighvolumetric energy density". J. Pre-Proof. June (2020).

3. Chenranyan Senthil, Chang Woo Lee. "Biomassderived biochar materials as sustainable energy sources for electrochemical energy storage devices". Renewable and Sustainable Energy Reviews. March (2021). Vol. 137, (110464).

4. Dacheng Zhang, Xiong Zhang, Xianzhong Sun, Haitao Zhang, Changhui Wang, Yanwei Ma. "High performance supercapacitor electrodes based on deoxygenated graphite oxide by ball milling". Electrochimica Acta. October (2013), Vol. 109, Pages (874-880).

5. Zohre Fahimi, Omran Moradlou. "Fabrication of ZnO@C foam: A flexible free-standing electrode for energy storage devices". Materials and Design. April (2020), Vol. 189, (108525).

6. Xiaolin $\mathrm{Xu}$, Jinrui Nana, Ju Wanga, Zepeng Gao. "Estimate of super capacitor's dynamic capacity". Energy Procedia. Vol. 105 (2017) (2194 - 2200).

7. M. Broseghini, M. D'Incau, L. Gelisio, N.M. Pugno. "Homogeneity of ball milled ceramic powders: Effect of jar shape and milling conditions". Data in Brief. February (2017), Vol. 10, Pages (186-191).

8. M. Broseghini, M. D'Incau, L. Gelisio, N. M. Pungo, p Scarddi. "Effect of jar shape on highenergy planetary ball milling efficiency: Simulations and Experiments". Materials and Design. November (2016), Vol. 110, Pages (365-374).

9. L S Godse, Vispi Karkaria, Dr P.B Karandikar, Dr N.R Kulkarni. "Innovative methods of ball milling to grind activated carbon as an electrode material for enhancing the performance of ultracapacitor". International Conference on Energy, Communication, Data Analytics and Soft Computing (ICECDS-2017).

10. Lars Huttermann, Roland Span, Pascal Maas, Viktor Scherer. "Investigation of a liquid air energy storage (LAES) system with different cryogenic heat storage devices". 10th International Conference on Applied Energy (ICAE2018), 22-25 August (2018), Hong Kong. Vol. 158, (4410-4415).

11. Tianhan Gao, Wei Lu. "Machine learning toward advanced energy storage devices and systems". A Cell Press Journal. December (2020), Vol. 24, Issue No. 1, (101936).

12. Vadahanambi Sridhar, Hyun Park. "Manganese nitride stabilized on reduced graphene oxide substrate for high performance sodium ion batteries, super-capacitors and EMI shielding". Journal of Alloys and Compounds. November (2019), vol. 808, (151748).

13. R. Jayendra Bharathi, Chintam Suresh Kumar. "Microwave processing and mechanical property evaluation of functionally graded materials with Al/SiC powders". Materials Today: Proceedings. (2018), Vol. 5, Pages (14481-14488).
14. Sithara P. Sreenilayam, Inam Ul Ahad, Valeria Nicolosi, Dermot Brabazon. "MXene materials based printed flexible devices for healthcare, biomedical and energy storage applications". Materials Today. March (2021), Vol. 43, Pages (99131).

15. Liu Yang, Hong Zheng, Lian Liu, Wenjie Wu, Shuya Wang. " $N$, O self-doped hierarchical porous carbon materials for high-performance supercapacitors". Results in Chemistry. Vol. 3, January (2021), (100109).

16. M. Broseghini, M. D’Incau, L. Gelisio, N.M. Pugno, P. Scardi. "Numerical and experimental investigations on new jar designs for high efficiency planetary ball milling". Advanced Powder Technology. July (2020), Vol. 31, Issue 7, Pages (2641-2649).

17. Xing Chen, Qun Liu, Tian Bai, Weiguo Wang, Faliang He, Meidan Ye. "Nickel and Cobalt Sulphide-Based Nanostructured Materials for Electrochemical Energy Storage Devices". Chemical Engineering Journal. Vol. 409, April (2021), (127237).

18. Xueying Kong, Shengyang Zhou, Maria Strømme, Chao Xu. "Redox active covalent organic framework-based conductive nanofibers for flexible energy storage device". Carbon. January (2021), Vol. 171, Pages (248-256).

19. Andrea Culcasi, Luigi Gurreri, Andrea Zaffora, Alessandro Cosenza, Alessandro Tamburini, Giorgio Micale. "On the modelling of an Acid/Base Flow Battery: An innovative electrical energy storage device based on $\mathrm{pH}$ and salinity gradients". Applied Energy. November (2020), Vol. 277, (115576).

20. Henry Kahimbi, Seok Bok Hong, MinHo Yang, Bong Gill Choi. "Simultaneous synthesis of $\mathrm{NiO} /$ reduced graphene oxide composites by ball milling using bulk $\mathrm{Ni}$ and graphite oxide for supercapacitor applications". Journal of Electroanalytical Chemistry. February (2017), Vol. 786, Pages (14-19).

21. L.S. Godse, Dr. P.B. Karandikar, Dr. M.Y. Khaladkar. "Study of Carbon Materials and Effect of Its Ball Milling, on Capacitance of Supercapacitor". Energy Procedia. (2014), Vol. 54, Pages (302 309).

22. Zhiwen Wang, Xiaotian Zhu, Peter. J. Deuss. "The effect of ball milling on birch, pine, reed, walnut shell enzymatic hydrolysis recalcitrance and the structure of the isolated residual enzyme lignin". Industrial Crops \& Products. September (2021), Vol. 167, (113493).

23. Daniele Sofia, Domenico Macri, Diego Barletta, Paola Lettieri, Massimo Poletto. "Use of titania powders in the laser sintering process: Link between process conditions and product mechanical properties". Powder Technology. March (2021), Vol. 381, Pages (181-188). 